\title{
Ethical Challenges in Clinical Research During the COVID-19 Pandemic
}

\author{
B.E. Bierer $(\mathbb{D} \cdot$ S.A. White • J.M. Barnes • L. Gelinas
}

Received: 16 August 2020 / Accepted: 10 September 2020

(C) Journal of Bioethical Inquiry Pty Ltd. 2020

\begin{abstract}
The sudden emergence of the COVID-19 pandemic brought global disruption to every aspect of society including healthcare, supply chain, the economy, and social interaction. Among the many emergent considerations were the safety and public health of the public, patients, essential workers, and healthcare professionals. In certain locations, clinical research was halted - or terminated - in deference to the immediate needs of patient care, and clinical trials focusing on the treatment and prevention of coronavirus infection were prioritized over studies focusing on other diseases.
\end{abstract}

B.E. Bierer $(\bowtie) \cdot$ S.A. White $\cdot$ J.M. Barnes $\cdot$ L. Gelinas

Multi-Regional Clinical Trials Center of the Brigham and Women's Hospital and Harvard, 14 Story Street, Cambridge, MA 02138, USA

e-mail: bbierer@bwh.harvard.edu

\section{B.E. Bierer}

Brigham and Women's Hospital and Harvard Medical School, 75 Francis Street, Boston, MA 02115, USA

\section{S.A. White}

Brigham and Women's Hospital, 75 Francis Street, Boston, MA 02115, USA

e-mail: sawhite@bwh.harvard.edu

\section{J.M. Barnes}

Ropes \& Gray, LLP, 800 Boylston Street, Boston, MA 02199, USA

e-mail: Mark.Barnes@ ropesgray.com

L. Gelinas

Advarra, 6940 Columbia Gateway Drive, Suite 110, Columbia, MD 21046, USA

e-mail: luke.gelinas@advarra.com
Difficult decisions were made rapidly; flexibility and reconsideration were necessary not only because the intensity and severity of infection varied over time and by locale but also because knowledge of the disease and understanding of its treatment (and prevention) grew. Here we discuss the ethical challenges in decisionmaking and competing ethical tensions during the pandemic in an effort to advance future preparedness.

Keywords COVID-19 $\cdot$ Pandemic $\cdot$ Bioethics $\cdot$ Clinical Research $\cdot$ Clinical Trials $\cdot$ Prioritization $\cdot$ Safety

Critical to the health of a population is the development and administration of safe and effective diagnostic, therapeutic, and vaccine products, processes that depend upon clinical trials as a part of well-organized product development programmes. The clinical research community was not prepared for the disruption caused by the COVID-19 pandemic, a force majeure that has impacted the operations and conduct of trials globally. Changes have been rapidly introduced to prioritize patient and participant (and healthcare worker) safety and to accommodate the requirement for social distancing to slow the spread of the virus. These changes - clinical trial suspensions, terminations, and continuationswere made after rapid consideration of whether risks could be mitigated and whether trial and data integrity could be assured. Here we examine the ethical challenges in decision-making during the pandemic in an effort to advance preparedness and learning. We analyse necessary trade-offs, differentiating decisions about 
ongoing clinical trials from decisions as to whether and when to initiate new studies.

In Part 1, we describe the emergence of the COVID-19 pandemic and immediate decisions regarding clinical trials. We consider the underlying ethical considerations in determining whether to continue, halt, or terminate an ongoing clinical trial, addressing the study objectives, criticality of treatment continuation for the study population, and potential accommodations in study conduct. In Part 2, the compromises and advantages of those accommodations are reviewed, highlighting participant privacy, risks, burdens, and sense of support. In Part 3, we discuss offering treatment options through expanded access programmes and clinical trials, using current examples to illustrate the importance of developing rigorous scientific evidence. We discuss the challenges of initiating and interpreting the results of trials to treat or prevent COVID19. We conclude with mandates for preparedness.

\section{COVID-19 Pandemic and Clinical trials}

Coronavirus Disease 2019 (COVID-19), the disease manifestation caused by the SARS-CoV-2, virus was first reported in December 2019 as an outbreak of pneumonia in Wuhan, China and rapidly spread throughout the world. On January 30, 2020, the World Health Organization (WHO) declared COVID-19 a Public Health Emergency of International Concern (World Health Organization 2020), and, on March 11, 2020, WHO declared it a pandemic (Branswell and Joseph 2020). As of May 12, 2020, there are over 4.2 million confirmed cases (COVID-19 Dashboard 2020) and over 290,000 deaths from the disease, and over 1300 clinical trials on "COVID-19" or "SARS-CoV-2" listed on Clinicaltrials.gov, of which 783 are interventional trials and seventeen are expanded access studies (ClinicalTrials.gov 2020). On May 1, 2020, the U.S. Food and Drug Administration (FDA) granted an Emergency Use Authorization (EUA) for remdesivir, a nucleoside ribonucleic acid (RNA) polymerase inhibitor, for the treatment of hospitalized patients with COVID19 (Remdesivir EUA Letter of Authorization 2020). Nevertheless, triaging trials, determining which trials to begin, continue, halt and resume, is an ongoing and evolving ethical challenge.

In the immediate aftermath of the declaration of the pandemic and, then, government guidance for social distancing as a public health measure to prevent or slow the spread of disease, trial sponsors, academic institutions, and investigators were required to determine whether ongoing trials should proceed (and if so, what modifications would need to be made), be suspended, or be discontinued altogether. These determinations were made considering several factors, including trial location, the indication for the trial, the urgency of continuation, the safety of participants and healthcare workers, and risks to trial integrity. Trial location is important, in that the pandemic affects regions differently and at different times. If a region is affected such that resources typically used for research must be redeployed to care for those with disease, priority must be given to trials of potential benefit. Those trials should continue only if the potential benefit in continuing the research exceeds the public health risks (including that of coming to the hospital or research site, and its potential for exposure, if necessary), and if there is sufficient staff to conduct the trials without sacrificing quality of clinical care. If a trial cannot be performed either because of staff unavailability or because the risk supersedes any possibility of benefit, the trial should be re-evaluated and likely suspended or discontinued. Should staff time be limiting, priority should be given to trials that evaluate potential treatment, prevention, or amelioration of COVID-19, and to trials dedicated to conditions of unmet medical need other than COVID-19 (e.g. oncology trials). If resource limitations prevent continuation of trials of potential therapies for serious or fatal diseases other than COVID-19, then those trials should be closed to further enrolment and preference should be given in continuing participants on study treatment who appear to be benefiting from the experimental therapy. Of course, changes to the protocol will likely require review and approval by the research ethics committee of record and may require securing an updated participant consent. Changes can be made prior to submission to the ethics committee with timely notification and follow-up if emergency changes to the protocol are required to promote participant (or staff) safety (Food and Drug Administration 2020).

Any consideration of changes to the protocol should consider potential modifications to study conduct that will enhance safety. Those changes include decreasing the number of protocol-required trial visits at any facility at which patients with COVID-19 are seen, increasing the number of remote visits (e.g., research home visits by a nurse or study team member; telehealth and/or video-enabled visits; local blood draws and imaging; remote, technology-enabled data capture); electronic 
informed consent (eConsent); and providing for home delivery of investigational products, among others.

\section{Risks, Burdens, and Benefits of Changes to Study Conduct}

While changes to study conduct may be necessary in order to enable the continuation or introduction of clinical trials, certain concerns with these potential changes must be addressed. Before any initiation of changes to the study protocol, consideration of confidentiality, privacy, data integrity, and safety must be addressed. Would a staff (e.g., visiting nurse) home visit compromise patient privacy or enlighten others living at home to the participant's condition? Would a home visit imperil personal safety of either the visiting staff or the participant? Is sufficient personal protective equipment available? If eConsent is suggested, can the identity of the signatory be confirmed? Can the data from remote visits be validated, especially if they depend on participant self-reports? How will source data be kept for remote visits? And are there visits that simply cannot be performed remotely due to the need to collect data from testing that cannot be done remotely or by home visit?

While there are some risks to remote visits, there are distinct advantages as well. Changes that optimize participant convenience, financial costs, and time are important and may even promote retention in the trial. No longer are participants spending significant time and effort traveling to the trial site to potentially wait in a crowded waiting room for a follow up visit that does not require onsite medical procedures or to have their blood drawn. Remote visits and data collection using certain mobile health technologies, even if part of a hybrid trial in which there are some in-person, clinic, or hospital visits interspersed with telehealth, are generally preferable to the participant and consistent with the principles of participant respect. Participants appear to appreciate the support that the visit and visiting staff member provides. Moreover, telehealth may decrease the threshold for the participant to engage outside of scheduled visits when necessary.

\section{Advancing Knowledge Through Expanded Access and Clinical Trials}

Knowledge about the protean manifestations of COVID-19 is developing rapidly; the virus appears to affect every organ system in the body and is expressed differently in different populations, varying by age, underlying condition, and region. This variability makes comparisons across populations and studies difficult, and even observational data can be interpreted differently depending on context. In the rush to redeploy resources to clinical care for acutely ill patients, there were few initial trials and still fewer randomized clinical trials (RCTs). Treatment consisted largely of symptomatic relief and was, unfortunately, often unsuccessful. In the United States and elsewhere, misinformation led to the promotion of certain therapeutic approaches for which little data existed. Foremost among treatments touted to be useful and potentially lifesaving, hydroxychloroquine, an oral drug approved for the treatment of malaria and lupus, was granted an EUA by the FDA despite the lack of data on efficacy (Facher 2020). The focus on hydroxychloroquine, in various dosages and with or without other therapies (e.g., azithromycin), prompted the rapid initiation of a number of trials. As of May 2020, there were approximately 170 hydroxychloroquine trials registered globally and about fifty in the United States. Many were underpowered, single site, and single arm studies, and likely many will fail to complete enrolment (Doughton 2020), in part because public interest in the treatment will wane prior to completion of the trial. Moreover, a published observational study failed to show benefit (Geleris et al. 2020), and there is concern for apparent cardiovascular toxicity (Chorin et al. 2020). As data emerged demonstrating lack of efficacy, the FDA withdrew the EUA.

The rapid rise and fall of enthusiasm for hydroxychloroquine illustrates a number of issues. First, the early and unfounded promotion of the drug as a promising treatment for COVID-19 led to public fervour for its therapeutic use, rendering an appropriate placebo ${ }^{1}$ controlled RCT difficult to enrol (Facher 2020; Sciama 2020). In the United States, President Trump's frequent mentions of hydroxychloroquine as a potentially efficacious therapy appeared to buttress and substantiate a therapeutic misconception, further exacerbating the difficulty with planning and completing rigorous evaluation of the drug in RCTs. Second, even if healthcare institutions and their investigators were committed to gathering evidence through RCTs, is it ethically appropriate to restrict access to an otherwise approved and marketed, relatively safe,

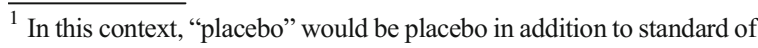
care.
} 
medication? If a healthcare institution decided that access would be limited to participation in an RCT, would the doctor be duty-bound to inform the patient that the drug is available elsewhere by prescription? Third, how does the fiduciary relationship between a patient and their doctor modify the boundaries between research and care? And lastly, while these are difficult issues, one major unfortunate consequence of facilitating off-label use, of course, is that future patients will not benefit from reliable evidence as to the efficacy and safety of hydroxychloroquine treatment or off-label treatment with other, already approved and marketed agents.

The proliferation of studies that are largely duplicative is impactful for other reasons as well. First, the number of trials alone increases the likelihood of a Type I error, a "false positive" (i.e., the incorrect rejection of a true null hypothesis), which could lead to further public confusion (or conviction) about the effectiveness of a therapy particularly if a false positive is reported before the others. Second, duplicative studies waste financial and human resources at a time when healthcare and research resources are limited. The resources used by duplicative trials may require delaying or foreclosing the initiation of trials that propose to study novel treatments. Even if those additional trials are nevertheless initiated, then patients will need to be allocated to one trial or another, and the process for that allocation is unclear. Should the patient be given the choice of all possible trials for which they are eligible? While technically possible, that task may be experienced as burdensome for the patient, and it assumes that the patient will have the knowledge base on which to make an informed decision. A multiplicity of open trials at an institution also risks the possibility that no trial will complete enrolment, that the number of patients in any institution will simply not support the successful execution of all studies (Gelinas et al. 2017). These considerations strongly support minimizing the number of currently enrolling studies at the same institution, especially when similar studies evaluating the same agent or intervention are undertaken elsewhere.

The development of evidence for treatment with the investigational agent remdesivir was more readily accomplished. Gilead Sciences Inc., the manufacturer of remdesivir, rigorously controlled access to the drug first through an expanded access programme and then in a number of RCTs of which two were open label and two were adaptive, randomized, double-blind, and placebocontrolled (Gilead Sciences Inc. 2020). The expanded access programme allowed the company to collect data and follow patients to determine outcome and adverse events. It did not have a comparator and therefore was of limited actionability. It is important to note that single-arm trials are more problematic in settings such as COVID-19, in which the disease course is largely unknown and/or changing, and the impact of co-morbidities is unclear compared to other epidemic infectious diseases such as Ebola, where the outcomes of historical controls were much better recognized.

At the same time as the single arm trials were underway, however, double-blind trials were also ongoing and reported only a short time later. One major trial, sponsored by the U.S. National Institutes of Health, demonstrated that treatment with remdesivir shortened the course of severe illness, although it did not modify the mortality rate (National Institute of Allergy and Infectious Disease 2020). On the basis of more rapid resolution of symptoms, the drug was granted an EUA for treatment of patients with COVID-19 (Remdesivir EUA Letter of Authorization 2020).

Given the demonstration of shortened symptomology but limited efficacy, are placebo-controlled trials still appropriate for hospitalized patients, or must all trials now include remdesivir either as the comparator to another intervention (remdesivir versus $\mathrm{X}$ ) or with and without the drug (remdesivir versus remdesivir plus $\mathrm{X}$ )? Must other trials now be stopped? Since this drug was granted an EUA for hospitalized patients with compromised oxygen saturation, how should treatment trials for other subgroups of patients be modified? Only through global communication and collaboration will common approaches be adopted and insights advanced.

\section{Knowledge and Preparedness}

Rapid progress depends upon research that is rigorous, of scientific and societal value, and executed at the highest standards of scientific validity, including blinding to treatment assignment, randomization, and controls (in this context either placebo or standard of care). These standards should not be put aside in the face 
of urgent and emergent situations (London and Kimmelman 2020). But society must balance the need for information and knowledge in the service of public health with the fiduciary responsibilities of the clinician to his or her patient and the patient's right to consent or not, concordant with their personal wishes.

We are only beginning to understand the nature of the COVID-19 disease and its different manifestations in different populations. The outcomes of trials conducted on severely ill individuals in the hospital — with hypoxemia, coagulopathy, and inflammatory reactions - will differ from those with mild or moderate disease and differ again from asymptomatic carriers. It is important to define the intended population and to develop common terms so that investigators, clinicians, and the public are able to communicate. In this regard, early efforts (CDISC 2020) to develop common data standards to enable data interoperability will ultimately save time and resources. In the absence of common data standards, the data from single-arm studies will be difficult to combine to derive usable information, and RCTs will be challenging to compare and contrast. Predefined platform trials represent an efficient approach to knowledge acquisition: the World Health Organization planned the platform trial SOLIDARITY, a trial that directly compares four treatments for COVID-19 (remdesivir, chloroquine and hydroxychloroquine, lopinavir-ritonavir, and lopinavir-ritonavir plus interferon-beta) (Kupferschmidt and Cohen 2020). While the specific treatments are perhaps less important (and can change), the model of a large adaptive platform trial, easy enrolment methods with clear eligibility requirements, minimal and necessary data collection, defined and robust endpoints, and global commitment to cooperation will expedite discovery.

As a medical and research community, remarkable progress has been achieved in a short time. But to expedite progress, we need leadership, preparation, and planning, and we must apply what we learn now to a "second wave" should one occur or, in its absence, to the next pandemic. As the wave of infectivity moves across countries, global collaboration is necessary to enable insights learned in one location to be applied to the next and to build upon knowledge, not reinvent it. For this to occur, common vocabularies and means of recording symptoms, co-morbidities, demographic and non-demographic characteristics of the individuals; agreement on common, objective endpoints and their definitions; collection of adverse safety events; electronic data capture and cloud computing; and commitments to share protocols, consents, and data must be in place, all of which may then be applied to rigorous research methodologies in the service of public health.

\section{References}

Branswell, H., and A. Joseph. 2020. WHO declared the coronavirus outbreak a pandemic. Stat News, March 11. https://www. statnews.com/2020/03/11/who-declares-the-coronavirusoutbreak-a-pandemic/. Accessed May 12, 2020.

CDISC. 2020. Interim user guide-COVID-19. Release date April 21. https://www.cdisc.org/standards/therapeutic-areas/covid19. Accessed May 12, 2020.

Chorin, E., M. Dai, E. Shulman, L. et. al. 2020. The QT interval in patients with COVID-19 treated with hydroxychloroquine and azithromycin. Nature Medicine 26: 808-809.

Clinical Trials.gov. 2020. https://clinicaltrials.gov/ct2 /results?cond=COVIDnucleoside ribonucleic acid (RNA) polymerase inhibitor $19 \&$ age $\_v=\&$ gndr $=\&$ type $=\& \mathrm{rslt}=$ \&Search=Apply. Accessed May 10, 2020.

COVID-19 Dashboard by the Center for Systems Science and Engineering (CSSE) at Johns Hopkins University (JHU). ArcGIS. Johns Hopkins University. 2020. https:/gisanddata. maps.arcgis.com/apps/opsdashboard/index.html\#/bda7594740 fd40299423467b48e9ecf6. Accessed May 9, 2020.

Doughton S. 2020. Clinical trial enrollment plummets as volunteers are scared off coronavirus drugs promoted by Trump. Seattle Times, May 9. https://www.seattletimes.com/seattlenews/health/clinical-trial-enrollment-plummets-asvolunteers-are-scared-off-coronavirus-treatment-pushed-bytrump/?utm_source=twitter\&utm_medium $=$ social \&utm campaign=article_inset_1.1. Accessed May 10, 2020.

Facher, L. 2020. Fact-checking Trump's claims about hydroxychloroquine the anti-malarial drug he's touting as a coronavirus treatment. STAT News, April 6. https://www. statnews.com/2020/04/06/trump-hydroxychloroquine-factcheck/. Accessed May 10, 2020.

Food and Drug Administration. 2020. FDA guidance on conduct of clinical trials of medical products during COVID-19 public health emergency. March 2020. Updated May 11, 2020. https://www.fda.gov/media/136238/download. Accessed May 12, 2020.

Geleris J., Y. Sun, J. Platt, et al. 2020. Observational study of hydroxchloroquine in hospitalized patients with Covid-19. New England Journal of Medicine 382: 2411-2418.

Gelinas L., H.F. Lynch, B.E. Bierer, and I.G. Cohen. 2017. When clinical trials compete: Prioritizing study recruitment. Journal of Medical Ethics 43(12): 803-809.

Gilead Sciences, Inc. 2020. Remdesivir clinical trials. https://www.gilead.com/purpose/advancing-global- 
health/covid-19/remdesivir-clinical-trials. Accessed May 12, 2020 .

Kupferschmidt, K., and J. Cohen. 2020. WHO launches global megatrial of the four most promising coronavirus treatments. March 22. https://www.sciencemag.org/news/2020/03/wholaunches-global-megatrial-four-most-promisingcoronavirus-treatments. Accessed May 11, 2020.

London, A.J., and J. Kimmelman. 2020. Against pandemic research exceptionalism. Science 368(6490): 476-477.

National Institute of Allergy and Infectious Disease, National Institute of Health. 2020. NIH clinical trial shows Remdesivir accelerates recovery from advanced COVID-19. April 29. https://www.niaid.nih.gov/newsevents/nih-clinical-trial-shows-remdesivir-acceleratesrecovery-advanced-covid-19. Accessed May 12, 2020.
Sciama, Y. 2020. Is Frances's president fueling the hype over an unproven coronavirus treatment? Science Magazine, April 9. https://www.sciencemag.org/news/2020/04/france-spresident-fueling-hype-over-unproven-coronavirustreatment. Accessed May 12, 2020.

Remdesivir EUA Letter of Authorization. 2020. https://www.fda. gov/media/137564/download. Accessed May 10, 2020.

World Health Organization. 2020. Coronavirus disease (COVID-19) outbreak. https://www.who. int/westernpacific/emergencies/covid-19. Accessed May 12, 2020.

Publisher's note Springer Nature remains neutral with regard to jurisdictional claims in published maps and institutional affiliations. 